Bio - grafia. Escritos sobre la Biología y su Enseñanza. ISSN 2027

Edición Extraordinaria. p.p. 1747-1757

Memorias del VIII Encuentro Nacional de Experiencias en Enseñanza de la Biología y la Educación Ambiental. III Congreso Nacional de Investigación en Enseñanza de la Biología.

\title{
Reflexiones acerca de la enseñanza de la influencia de la alimentación en la evolución humana, en niños de quinto de primaria
}

\section{Reflections on the teaching of the influence of diet in human evolution, in fifth grade children}

\author{
Rátiva Avella Yudy Cecilia'.
}

\section{Resumen}

Teniendo en cuenta los problemas mundiales que se presentan debido a la malnutrición; la alimentación y la nutrición humana, constituyen temáticas de vital importancia en la enseñanza que se realiza a los niños de primaria, a temprana edad se deben brindar los conocimientos necesarios para poder influir en sus comportamientos alimenticios en la edad adulta; por lo tanto se hacen necesarios procesos de reflexión docente afín de contribuir a mejorar las prácticas pedagógicas. En este artículo se presenta una reflexión sobre la importancia de desarrollar en el aula de clase de los niños de quinto de primaria la enseñanza de la alimentación desde una perspectiva histórica y biológica teniendo en cuenta la evolución humana y la alimentación hoy, esta surge después de un proceso de reflexión y análisis de la práctica pedagógica, efectuada al analizar las respuestas proporcionadas al cuestionario semiestructurado Representación del Contenido (ReCo), los resultados obtenidos establecen la importancia de implementar procesos de reflexión docente que permitan implementar diferentes estrategias de enseñanza mediante las cuales se aborde temáticas socialmente relevantes desde diferentes perspectivas.

Palabras claves: alimentación y nutrición humana, evolución humana, Representación de Contenido ( $\operatorname{ReCo}$ ), reflexión docente, enseñanza.

\section{Abstract}

Taking into account the global problems that arise due to malnutrition, food and human nutrition, these are topics of vital importance in what is taught to children in primary school, because early knowledge required to influence eating behavior in

${ }^{1}$ Docente IED San Isidro SO, estudiante de maestría en gestión de tecnología educativa, Universidad de Santander UDES. yucera@hotmail.com 
Bio - grafia. Escritos sobre la Biología y su Enseñanza. ISSN 2027

Edición Extraordinaria. p.p. $1747-1757$

Memorias del VIII Encuentro Nacional de Experiencias en Enseñanza de la Biología y la Educación Ambiental. III Congreso Nacional de Investigación en Enseñanza de la Biología.

adulthood must be provided; therefore teaching reflection processes in order to help improve teaching practices are necessary. This article reflects on the importance of developing in the classroom of children in fifth grade teaching power from a historical and biological perspective considering human evolution and food today, this comes after a process of reflection and analysis of pedagogical practice, conducted after analyzing the replies provided to the semi-structured questionnaire Representation of Content ( $\operatorname{ReC}$ ), the results establish the importance of generating reflection processes on teaching allowing to implement different teaching strategies through which to address socially relevant issues from different perspectives.

Key words: food and human nutrition, human evolution, Content Representation $(\mathrm{ReCO})$, teaching reflection, teaching.

Los procesos de reflexión docente sobre su práctica pedagógica constituyen acciones que permiten cuestionarse, actualizarse y elaborar diferentes estrategias pedagógicas, que favorezcan el aprendizaje de los estudiantes.

Muchas veces estos se efectúan cuando se observa que lo aprendido por lo niños no permite responder adecuadamente a los problemas que se presentan en la vida cotidiana, lo cual puede llegar a generar problemas socialmente relevantes; como es el caso mala alimentación y la malnutrición. Frente a los esfuerzos que realiza el profesor cotidianamente en el aula de clases, en la cual los estudiantes evidencian diferentes niveles de aprendizaje sobre la alimentación y la nutrición humana, surge la desazón cuando en los descansos escolares, en casa o en su diario vivir las prácticas alimenticias de los estudiantes no son adecuadas, algunas veces llegando a la adultez con problemas generados por la mala alimentación.

También existe esta reflexión cuando el docente desea mejorar su práctica pedagógica, en un proceso de cualificación que al ser constante y permanente influirá positivamente en su desarrollo personal y profesional, tal y como lo afirma Castellanos y Yaya (2013) "el ejercicio reflexivo sobre la propia práctica de enseñanza a la luz del conocimiento pedagógico puede contribuir a que los docentes en formación revisen 
Bio - grafia. Escritos sobre la Biología y su Enseñanza. ISSN 2027

Edición Extraordinaria. p.p. $1747-1757$

Memorias del VIII Encuentro Nacional de Experiencias en Enseñanza de la Biología y la Educación Ambiental. III Congreso Nacional de Investigación en Enseñanza de la Biología.

críticamente su trabajo, puedan explicar sus propias acciones y reorienten sus prácticas pedagógicas" (p .22).

Algunas propuestas mediante las cuales se pueden llevar a cabo procesos de reflexión docente, incluyen: entrevistas de autorreflexión; acompañamiento entre pares, durante el cual se analizan diversas prácticas pedagógicas; la documentación narrativa de prácticas escolares, permite reconstruir, interpretar las acciones docentes cuando escriben, leen, reflexionan y conversan entre colegas acerca de sus propias experiencias educativas (Suárez, 2007); biografías profesionales, permiten analizar la influencia de la historia de vida de los profesores en su desempeño profesional, cómo obstaculiza o favorece, entre otras. En este artículo se propone el uso de los cuestionarios semiestructurados de las Representaciones de Contenido (ReCo), como estrategias que permitirán que el profesor reflexione y analice su práctica pedagógica cuando enseña un contenido específico a los estudiantes.

La ReCo es un instrumento desarrollado por Loughran, Milroy, Gunstone y Mulhall (2001), que permite evidenciar la forma en que un profesor enseña un contenido específico y explicar el por qué lo enseña así, en este caso permite reflexionar sobre la enseñanza de la alimentación y la nutrición humana.

La enseñanza de estos contenidos a estudiantes de primaria constituye un gran reto; al ser una actividad cotidiana, influenciada en gran parte por la perspectiva cultural: las prácticas alimenticias sociales y familiares, su situación económica, entre otros. Los estudiantes llegan al aula de clase con conocimientos previos que algunas veces obstaculiza el aprendizaje de los estudiantes; además, no solo se debe instruir a los estudiantes con temáticas propias de la biología; sino que se debe comenzar a desarrollar en los niños actitudes reflexivas sobre la importancia de una alimentación balanceada y saludable, este aspecto adquiere una gran importancia en el mundo actual, donde las grandes multinacionales de la alimentación utilizan la publicidad para atraer a las personas para que consuman golosinas y comida rápidas. Además la reflexión es muy importante ante la percepción que gran parte de la población tiende a la uniformidad en la alimentación, desconociendo ciertos alimentos y muchas veces olvidando aquellas comidas autóctonas, propias de su cultura y su región, tal como lo señala Bahamonte (2009): 
Bio - grafia. Escritos sobre la Biología y su Enseñanza. ISSN 2027

Edición Extraordinaria. p.p. 1747-1757

Memorias del VIII Encuentro Nacional de Experiencias en Enseñanza de la Biología y la Educación Ambiental. III Congreso Nacional de Investigación en Enseñanza de la Biología.

La alimentación es un fenómeno complejo que integra estrechamente las dimensiones biológica y cultural, al punto de condicionarlas mutuamente. El hecho alimentario, en nuestras sociedades atravesadas por el fenómeno de la globalización, requiere una aproximación multidimensional, que visualice el estudio de la alimentación en el marco de una cultura. (p.4)

La enseñanza de la alimentación y la nutrición debe generar aprendizajes significativos que incidan en la vida futura y en la salud de los estudiantes, sus familias y la comunidad.

\section{Metodología}

En el marco de un convenio de cooperación entre el Instituto de Investigaciones pedagógicas (IDEP) y la Universidad Pedagógica Nacional (UPN), grupo de investigación el Conocimiento Profesional del Profesor de Ciencias, se realiza la convocatoria "El profesor de Ciencias como sujeto de conocimiento. Reflexionemos y sistematicemos", para profesores interesados en reflexionar sobre sus experiencias pedagógicas relacionadas con la alimentación y la nutrición humana, mediante estrategias de apoyo e intercambio de saberes. Convocatoria a la cual la autora de este artículo se inscribe.

En un primer momento se realiza un acompañamiento de todas las clases mientras se realiza la enseñanza de la alimentación y la nutrición humana y después, se realizan actividades de reflexión, entre ellas: se analizan las respuestas dadas a un cuestionario semiestructurado de Representación del Contenido ( $\operatorname{ReCo}$ ), instrumento modificado y validado por Dueñas y Valbuena ${ }^{2}$, éste consta de 15 preguntas relacionadas con la enseñanza de este contenido. En este artículo se analizará solamente la respuesta dada a la pregunta 12: Cuando enseña este tema, ¿tiene en cuenta aspectos históricos, epistemológicos? Si su respuesta es afirmativa explique (por qué, cómo, qué).

\footnotetext{
2 Instrumento adaptado y validado por Dueñas, A.M y Valbuena, É., a partir de Loughran, J., Milroy, P., Gunstone, R. y Mulhall, P. (2001). Documenting Science Teachers' Pedagogical Content Knowledge Trough PaP-eRs. Research in Science Education. 31: 289-307
} 
Bio - grafia. Escritos sobre la Biología y su Enseñanza. ISSN 2027

Edición Extraordinaria. p.p. 1747-1757

Memorias del VIII Encuentro Nacional de Experiencias en Enseñanza de la Biología y la Educación Ambiental. III Congreso Nacional de Investigación en Enseñanza de la Biología.

\section{Resultados}

Los procesos de reflexión a nivel de la enseñanza de la alimentación y nutrición humana permiten cuestionarme sobre qué puedo hacer para que el aprendizaje que efectúan los alumnos sea más efectivo, para que ellos en su vida cotidiana sean capaces de poner en práctica lo aprendido en clase; específicamente al reflexionar sobre la respuesta dada a la pregunta 12 del cuestionario ReCo, en la cual indico que no tengo en cuenta éstos aspectos en su enseñanza, debido al desconocimiento y a que no había pensado en la relevancia que puede tener el desarrollar estos contenidos con los estudiantes de primaria.

Generalmente durante la enseñanza que brindo sobre la nutrición en estos grados, les hablo de la importancia de los alimentos, su clasificación, la alimentación balanceada, de dónde se obtienen los alimentos, la digestión, la anatomía y fisiología del sistema digestivo, entre otros. Pero no doy importancia a los aspectos históricos, ni epistemológicos. Adicionalmente los libros que tengo de guía tampoco hacen referencia a este aspecto, por lo que no había pensado en la posibilidad de incluirlo durante la enseñanza de esta temática.

Este escrito no pretende hacer un recorrido por la historia y la epistemología de la alimentación y la nutrición humana, sino que es una reflexión sobre la enseñanza de la historia de la alimentación y cómo se puede relacionar con la enseñanza de éste conocimiento biológico en niños de quinto de primaria.

Al estar la alimentación y la nutrición humana inmersas en la cotidianidad, en la cultura de una población, en la economía, entre otros, pienso que su enseñanza no se puede limitar únicamente a aspectos biológicos; al respecto, observo que la mayoría de las veces los niños y jóvenes saben cuáles son los componentes nutricionales de cierta dieta, conocen qué alimentos son adecuados y cuáles son perjudiciales para su salud, lo que indica que poseen el conocimiento biológico adecuado; pero este no se refleja en su comportamiento alimentario, lo cual indica que se deben fortalecer otros tipos de conocimientos para tratar de dar una enseñanza más integral, en la que se considere la alimentación y la nutrición desde diferentes campos del conocimiento.

El objetivo principal es incidir en las practicas alimenticias de los estudiantes, para lo cual analizaré la importancia de desarrollar en el aula de clase de los niños de quinto de primaria la enseñanza de la alimentación desde una perspectiva histórica y biológica 
Bio - grafia. Escritos sobre la Biología y su Enseñanza. ISSN 2027

Edición Extraordinaria. p.p. $1747-1757$

Memorias del VIII Encuentro Nacional de Experiencias en Enseñanza de la Biología y la Educación Ambiental. III Congreso Nacional de Investigación en Enseñanza de la Biología.

teniendo en cuenta la evolución humana y la alimentación hoy, con lo cual trataré de dar respuesta a los siguientes interrogantes: cómo puede la historia de la alimentación influir en el comportamiento de los estudiantes, cuáles son los conocimientos relacionados con la alimentación y la evolución que se deben desarrollar, cuál es la importancia de su enseñanza.

Surgen entonces los siguientes interrogantes ¿Es apropiado tratar la temática de la evolución en niños de primaria? ¿Qué nivel de profundidad se deben tratar en clase? ¿Cómo puedo relacionar la evolución humana con la alimentación hoy? Para tratar de responder estos cuestionamientos, realicé una revisión bibliográfica al respecto, en la cual se evidencia y resalta la importancia de tratar los conocimientos más elementales de la evolución de la tierra y los seres vivos con los niños desde temprana edad, de forma tal que ellos puedan comenzar a construir explicaciones cercanas al conocimiento científico escolar, se reconoce la importancia de enseñanza de la evolución como eje central en la comprensión y adecuada conceptualización de la diversidad biológica, estructuras anatómicas, procesos fisiológicos y bioquímicos, entre otros. Al respecto Mesa y Buitrago (2014), sugieren:

Que el pensamiento evolutivo se comience a desarrollar desde los primeros grados de educación básica primaria y media; sobre todos porque los retos actuales demandan de una reflexión sobre las dinámicas de la naturaleza, la importancia de la conservación de las especies, y nuestro papel ético y responsable con la ciencia, tecnología y sociedad. (p. 41)

A pesar de su importancia, se reconoce la dificultad de que esta temática se trate a nivel primario porque no se reconoce la necesidad de que los estudiantes vayan construyendo su conocimiento sobre este tema y en algunas ocasiones a causa de la dificultad que representa tratarlo debido a las concepciones que poseen los estudiantes generalmente de tipo religioso.

Teniendo en cuenta la propuesta de Cañal (2009), se presentan las ideas que deben construirse progresivamente de forma elemental durante los estudios primarios: ¿Cuál es el origen de la vida y de los seres vivos en la Tierra?, ¿Han cambiado los seres vivos a lo largo de la historia de la Tierra?, ¿Qué son las especies? ¿Cómo se forman, cambian y extinguen las especies?, ¿Cómo se ha producido la evolución de los seres vivos en la Tierra? y ¿Cómo se ha producido la evolución de los seres humanos?. Además propone que de acuerdo a las 
Bio - grafia. Escritos sobre la Biología y su Enseñanza. ISSN 2027

Edición Extraordinaria. p.p. 1747-1757

Memorias del VIII Encuentro Nacional de Experiencias en Enseñanza de la Biología y la Educación Ambiental. III Congreso Nacional de Investigación en Enseñanza de la Biología.

edades, considera que una opción didáctica basada en la investigación escolar que tenga en cuenta los intereses de los estudiantes sería la adecuada para desarrollar la temática.

Si se tienen en cuenta que los estudios de quinto de primaria se desarrollara la pregunta ¿Cómo se ha producido la evolución de los seres humanos?, la reflexión que surge es cómo puedo relacionar durante la enseñanza la importancia que ha tenido la alimentación en la hominización. De acuerdo con Leonard (2009), "los cambios operados en el régimen alimentario han construido una fuerza motriz de la evolución humana" (p. 49). Las principales características de los homínidos incluyen el bipedismo, su postura erguida, el tamaño de su caja craneana, se asocian a procesos de selección natural relacionados con la calidad de la alimentación y el rendimiento energético.

En los inicios de la civilización la alimentación puede ser vista como un acto de vital importancia que suple necesidades básicas de supervivencia, al mismo tiempo se relaciona el desarrollo fisiológico con la alimentación. Se pueden reflexionar de manera básica acerca del rol que jugó la alimentación:

1. Desde el Australopithecus robustus al Austrolopithecus africanus, la importancia del bipedismo, cómo permitió el transporte y consecución de los alimentos. Además de qué forma influyó en la alimentación el desarrollo de herramientas y la caza de animales. Además este tipo de locomoción resulta desde el punto de vista energético menos costosa que la cuadrúpeda. Se debe tener en cuenta que la evolución de los primeros homínidos ocurrió en bosques abiertos y praderas, donde se debe gastar bastante energía para la consecución de alimentos. Es importante recalcar en este aspecto, ya que el gasto de energía era muy alto debido a las dificultades en su consecución y al gasto que representaba la caza, por lo tanto cualquier ahorro de energía representaba una ventaja evolutiva ya que permitía cuidar las crías y dedicarlas a la reproducción.

2. En el Austrolopithecus africanus (grácil), Homo habilis, Homo erectus, Homo sapiens, la importancia del desarrollo del tamaño de la caja craneana y la mayor proporción de la masa cerebral, lo que ocasionó un gran aumento en el gasto de energía y esto fue posible al cambió a una dieta rica en calorías y nutrientes. 
Bio - grafia. Escritos sobre la Biología y su Enseñanza. ISSN 2027

Edición Extraordinaria. p.p. 1747-1757

Memorias del VIII Encuentro Nacional de Experiencias en Enseñanza de la Biología y la Educación Ambiental. III Congreso Nacional de Investigación en Enseñanza de la Biología.

También, la evolución de la anatomía de la mano, permitiría conseguir los alimentos de una forma más precisa y ágil, mejorando la dieta que se consumía. Un aspecto muy importante también fue la aparición del fuego con el Homo erectus y cómo este descubrimiento revolucionaria la alimentación humana al poder preparar y cocinar los alimentos, que no eran aptos para el consumo en su estado natural. Además analizar la importancia de la consecución de los alimentos, la caza y la alimentación en el establecimiento de las relaciones sociales.

La mejora en la calidad de la alimentación con mayor aporte de nutrientes y calorías ha resultado eficaz para el ser humano actualmente este tendencia continua, se están generando alimento hipercalóricos, hipervitamínicos, con mayor aporte de nutrientes, incluso las plantas están siendo modificadas genéticamente para tal fin; sin embargo, hay que tener en cuenta que las dinámicas de nuestros antepasados eran muy activas, ellos gastaban bastante energía en adquirir los alimentos y en sobrevivir; actualmente la mayoría de los seres humanos son más sedentarios y si estos alimentos hipercalóricos se consumen en exceso, pueden traer como consecuencia que aumenten los casos de sobrepeso y obesidad. Tal como lo afirma Leonard (2009): "somos las victimas de nuestro éxito evolutivo hemos adquirido una dieta muy calórica, mientras minimizábamos la cantidad de energía que gastamos en nuestra actividad diaria" (p.57).

Además, este proceso se ve agudizado en algunas regiones del mundo se observan procesos de aculturización alimentaria debido principalmente a la globalización, cuando la identidad cultural de una población es débil y no existe un conocimiento sobre los alimentos autóctonos, sus virtudes y su importancia en la cocina tradicional, es más fácil sustituirlos y adoptar una cultura alimenticia foránea representada por las comidas rápidas o también denominada comida chatarra. Al respecto se puede reflexionar con los estudiantes sobre: ¿Cómo influye el consumo de comidas rápidas y el sedentarismo en la salud de los individuos? Y deben desarrollar actividades que permitan asumir una actitud crítica, frente a la publicidad alimentaria, que incita a consumir este tipo de alimentos

\section{Conclusiones}

Los procesos de reflexión docente, constituyen valiosas herramientas que permiten

que actualicen sus conocimientos y mejoren sus prácticas pedagógicas, tal como lo afirma 
Bio - grafia. Escritos sobre la Biología y su Enseñanza. ISSN 2027

Edición Extraordinaria. p.p. 1747-1757

Memorias del VIII Encuentro Nacional de Experiencias en Enseñanza de la Biología y la Educación Ambiental. III Congreso Nacional de Investigación en Enseñanza de la Biología.

Anijovich (2005): "En la actualidad hay consenso que la reflexión guía el crecimiento profesional, estimula la construcción de conocimientos constituyéndose en una estrategia formativa" (p.22).

La experiencia realizada permite demostrar que el cuestionario ReCo, puede ser utilizado para la reflexión docente, ayudando a crear estrategias mediante las cuales mejore la enseñanza de un contenido específico en este caso de la alimentación humana, lo cual se verá reflejado en el aprendizaje de los estudiantes.

El conocimiento relacionado con la alimentación humana, está íntimamente relacionado con la evolución y adaptación del ser humano. De acuerdo con Martínez y Rodríguez (2002):

Con el estudio de la evolución de la alimentación se puede tener una visión de por qué y cómo ingerimos determinados alimentos y a la vez facilita la comprensión de cómo intentamos satisfacer ciertas necesidades, no sólo fisiológicas, a través de la ingesta de alimentos. En este recorrido por todas las épocas se observan los momentos o hitos históricos que han dejado una impronta en nuestras costumbres gastronómicas, pues en cada etapa de la historia se producen acontecimientos que quedan reflejados también en la mesa. (p.80)

Al fortalecer el conocimiento sobre la historia de la alimentación, se puede enseñar a los estudiantes la importancia que ha tenido el hecho alimentario, la contribución de las distintas culturas, se tratará de relacionar con aspectos biológicos por ejemplo: la evolución humana, se analizarán aspectos alimentarios y nutricionales desde diferentes campos de conocimientos para tratar de dar respuesta a los fenómenos alimentarios que se presentan en la sociedad actual.

Se espera que mediante la enseñanza de la alimentación desde una perspectiva histórica y biológica relacionada con la evolución humana, se puedan desarrollar competencias en los estudiantes que le permitan tener una actitud reflexiva y crítica frente al sedentarismo, al exceso de consumo de alimentos hipercalóricos y a la publicidad generada por las grandes multinacionales de alimentos que promueven el consumo de sus 
Bio - grafia. Escritos sobre la Biología y su Enseñanza. ISSN 2027

Edición Extraordinaria. p.p. 1747-1757

Memorias del VIII Encuentro Nacional de Experiencias en Enseñanza de la Biología y la Educación Ambiental. III Congreso Nacional de Investigación en Enseñanza de la Biología.

productos caracterizados por un alto contenido de azúcares, grasas, cuyo consumo frecuente puede generar problemas de salud.

\section{Referencias Bibliográficas}

Anijovich, R. (2005). La reflexión como estrategia para el desarrollo profesional de los docentes, En: Formación de Profesionales Reflexivos en Diseño y Comunicación. XIII Jornadas de Reflexión Académica en Diseño y Comunicación (pp. 22-23). Buenos Aires, Argentina: Editor Estela Pagani.

Bahamonte, N. (2009). Un enfoque para la educación alimentaria en la escuela. En: Educación alimentaria y nutricional. Ministerio de Educación, Presidencia de la Nación Argentina (pp.1-128). Buenos Aires, Argentina.

Cañal, P. (2009). Acerca de la enseñanza sobre la evolución biológica en la escuela infantil y primaria. Alambique. Didáctica de las ciencias experimentales. 62, 75-91.

Castellanos, S., y Yaya., R. (2012). La reflexión docente y la construcción de conocimiento: una experiencia desde la práctica, revista electrónica de educación "sinectica", 41. 1-18. Recuperado de http://www.sinectica.iteso.mx/ articulo/?id=41_la_reflexion_docente_y_la_construccion_de_conocimiento_ una_experiencia_desde_la_practica

Leonard, W. (2003). Incidencia de la dieta en la hominización. Revista Investigación y Ciencia. 317, 49-57.

Martínez, C., y Rodríguez, A. (2002). Influencia de la alimentación en el comportamiento humano a través de la historia, OFFARM, Elsevier. 21 (7), 80-88.

Martínez-Sierra, G., y Poitier, P. (2008). Una epistemología histórica del producto vectorial: Del cuaternión al análisis vectorial. Am. J. Phys. Educ. 2 (2), 201-208.

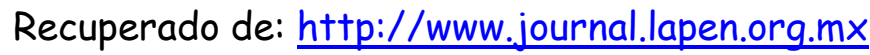


Bio - grafia. Escritos sobre la Biología y su Enseñanza. ISSN 2027

Edición Extraordinaria. p.p. $1747-1757$

Memorias del VIII Encuentro Nacional de Experiencias en Enseñanza de la Biología y la Educación Ambiental. III Congreso Nacional de Investigación en Enseñanza de la Biología.

Mesa, J., y Buitrago, J. (2014). Una resignificación para la enseñanza del concepto de evolución biológica desde un análisis histórico y epistemológico de la perspectiva de Darwin (tesis de pregrado). Universidad de Antioquia, Medellín, Colombia.

Suárez, H. (2007). Docentes, narrativas e indagación pedagógica del mundo escolar. Hacia otra política de conocimiento para la formación docente y la transformación democrática de la escuela. e- Eccleston. Formación Docente. 3 (7), 1-30. 